\title{
LESSONS LEARNED FROM FLIGHT OBSERVATIONS OF THE GOES-R MAGNETOMETER
}

\author{
R. Schnurr ${ }^{1}$, T. Bonalsky ${ }^{2}$, M. Todirita ${ }^{3}$, J. Kronenwetter ${ }^{4}$, D. Early ${ }^{5}$, M. Grotenhuis ${ }^{6}$, R. Studer ${ }^{7}$, \\ D. Carter ${ }^{8}$, R. Dence ${ }^{9}$, M. Wolf ${ }^{10}$, J. Mandi ${ }^{11}$ \\ ${ }^{1}$ NASA, Code 560, GSFC, 20771, USA, richard.g.schnurr@nasa.gov \\ ${ }^{2}$ NASA, Code 549, GSFC, 20771, USA, todd.m.bonalsky@nasa.gov \\ ${ }^{3}$ NOAA, Code 417, GSFC, 20771, USA, monica.todirita-1@nasa.gov \\ ${ }^{4}$ Chesapeake Aerospace, Code 417, GSFC, 20771, USA, jeffrey.a.kronenwetter@nasa.gov \\ ${ }^{5}$ Chesapeake Aerospace, Code 417, GSFC, 20771, USA, derrick.a.early@nasa.gov \\ ${ }^{6}$ SSAI, Code 417, GSFC, 20771, USA, michael.g.grotenhuis@nasa.gov \\ ${ }^{7}$ AS and D, Code 417, GSFC, 20771, USA, robert.j.studer@nasa.gov \\ ${ }^{8}$ Thearality, Inc, Code 417, GSFC, 20771, USA, delano.r.carter@nasa.gov \\ ${ }^{9}$ Lockheed Martin Space, Littleton, CO, USA, robert.j.dence@lmco.com \\ ${ }^{10}$ Lockheed Martin Space, Littleton, CO, USA, molly.kathleen.wolf@lmco.com \\ ${ }^{11}$ Lockheed Martin Space, Littleton, CO, USA, joseph.l.mandi@Imco.com
}

\begin{abstract}
The The Geostationary Operational Environmental Satellite (GOES)-16 observatory was launched on 19 November 2016. During daily on-orbit operations, shadowing of the inboard magnetometer sensor unit occurs due to spacecraft geometry and solar angle. Throughout the shadowing periods of the inboard magnetometer, anomalous excursions of 20 nanotesla (nT) are observed. In addition to the excursions during shadow events, the measurement difference between the inboard and outboard magnetometer varies over the day, indicating erroneous measurements by one or both magnetometers. In addition, based on the deployment rotations, the zero offsets of the $\mathrm{X}$ and $\mathrm{Y}$ axes were found to be significantly different, $\sim 30 \mathrm{nT}$, from ground calibration data.
\end{abstract}

Because of these observations, an extensive root cause investigation was undertaken to correct the magnetometer system for the next spacecraft in the GOES-R series. This paper documents the efforts of that activity and the lessons learned as a result of the investigation.

\section{INTRODUCTION}

The Geostationary Operational Environmental Satellite (GOES)-16 observatory, which is part of the National Oceanic and Atmospheric Administration (NOAA) GOES-R Series constellation of weather satellites, was launched on 19 November 2016. The GOES-16 Magnetometer System includes two boom-mounted fluxgate sensors 6.3 meters and 8.5 meters from the spacecraft as shown in Fig. 2. The inboard magnetometer is mounted facing toward the spacecraft, while the outboard magnetometer faces away from the spacecraft. The GOES-16 magnetometers post-launch testing and checkout began on 7 December 2016 during magnetometer boom deployment. During daily on-orbit operations, shadowing of the inboard magnetometer sensor unit occurs due to spacecraft geometry and solar angle. Throughout the shadowing periods of the inboard magnetometer, anomalous excursions of 20 nanotesla (nT) are observed. In addition to the excursions during shadow events, the measurement difference between the inboard and outboard magnetometer varies over the day, indicating erroneous measurements by one or both magnetometers. Fig. 2 shows the variation in the difference between the inboard and outboard magnetometers over multiple days. For a nominal system the difference between the two magnetometers should be small $(<2 \mathrm{nT})$ with small variances in the spacecraft field causing diurnal variation [1].

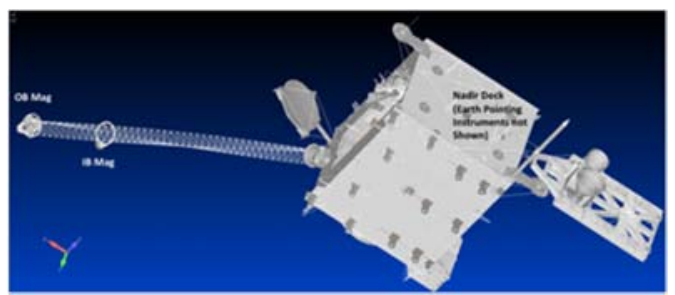

Figure 1. GOES-16 Observatory depicting Magnetometer Boom and Magnetometers

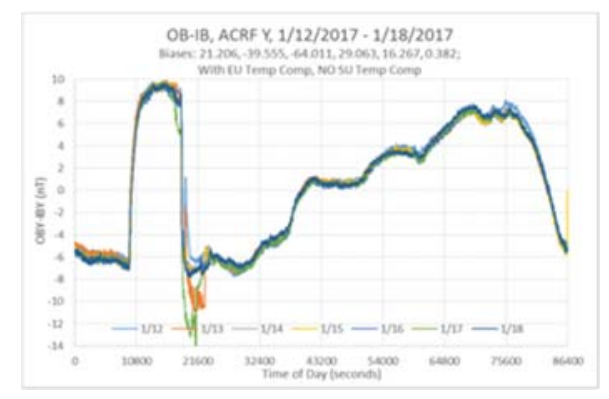


Figure 2. Daily trend of anomalous signature over 1 week period

Furthermore, based on the deployment rotations, the zero offsets of the $\mathrm{X}$ and $\mathrm{Y}$ axes on both inboard and outboard magnetometers were found to be significantly different, $\sim 30 \mathrm{nT}$, from ground calibration data as shown in Fig. 3. Subsequent calibration maneuvers confirmed the large zero offsets in all three axes.

A comparison of both the inboard and outboard magnetometers to other on-orbit GOES (GOES-13, GOES-14, and GOES-15) shows the diurnal variation in the differences is not solely due to erroneous inboard magnetometer readings. Fig. 4 shows the outboard magnetometer has a diurnal error variation of $+/-5 \mathrm{nT}$ as compared to the GOES-13 outboard magnetometer. [2][3]. The comparison was performed when GOES-16 was collocated with GOES-13.
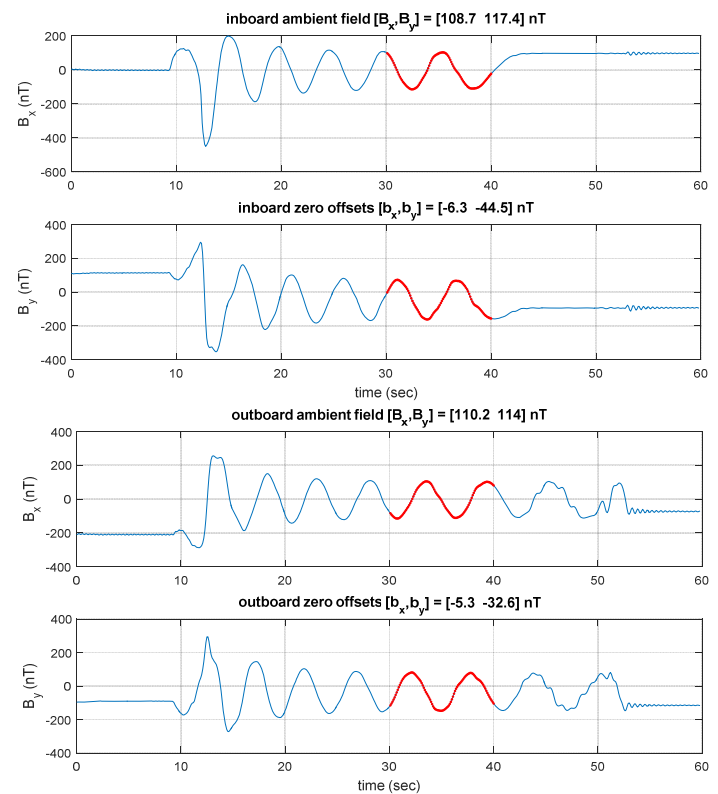

Figure 3 Inboard and Outboard Magnetometer $X$ and $Y$ Axes during deployment

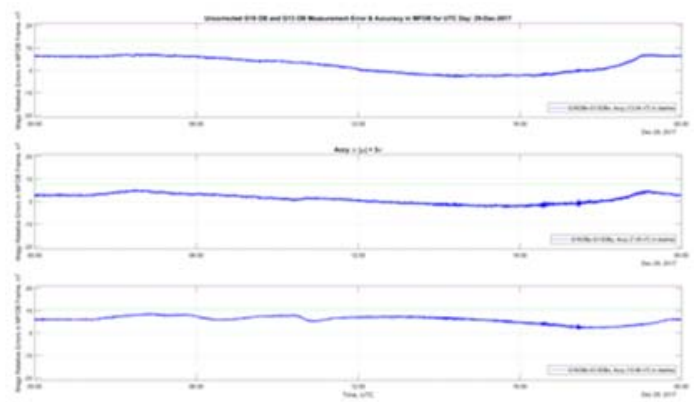

Figure 4 GOES-16 wrt GOES-13 Magnetometer Measurement Diurnal Error Curves

\section{ROOT CAUSE INVESTIGATION}

As stated, the large $20 \mathrm{nT}$ rapid excursions in the inboard magnetometer are correlated to shadowing of the inboard magnetometer by the spacecraft. Figs. 5 through 7 show the inboard being shadowed by the spacecraft body and subsequently by the high gain antenna wing [2].

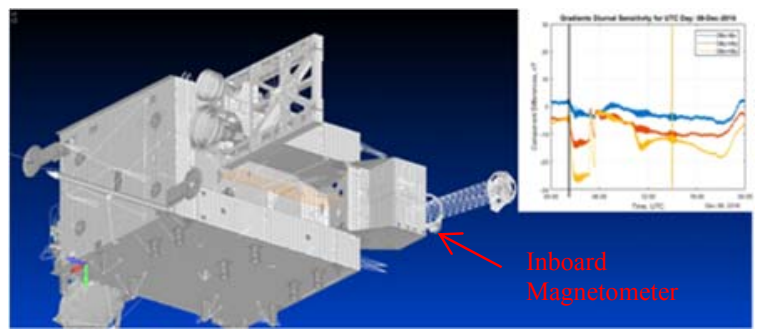

Figure 5 Start of GOES-16 inboard Magnetometer Shadowing by Observatory

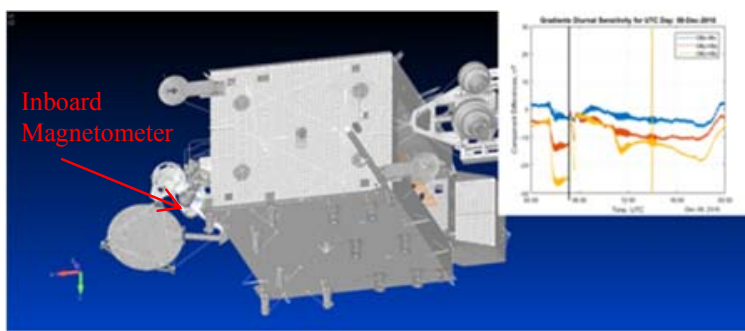

Figure 6 End of GOES-16 inboard Magnetometer Shadowing by Observatory

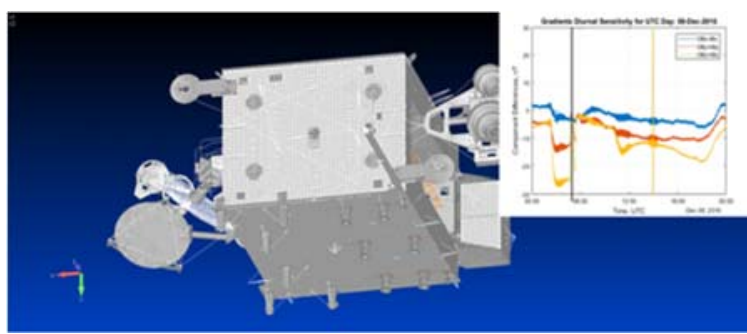

Figure 7 Start of GOES-16 inboard Magnetometer Shadowing by High Gain Antenna Wing

The two most likely root causes of the erroneous large difference between inboard and outboard magnetometer measurements were determined to be either a thermoelectric effect (Seebeck or eddy currents) generating a current loop, or unintended contamination from magnetic material [4][5]. Several potential sources for the Seebeck effect were identified and tests were performed on spare parts to attempt to replicate the magnetic signature. The potential sources of Seebeck or eddy currents are:

- The harness p-clamps wrapped in copper tape in contact with vapor deposited aluminum (VDA) on the magnetometer sensor epoxy composite mounting plate creating the possibility of voltage difference along the loop formed by the 
harness, cooper tape, and VDA. Also, the copper tape in contact with the harness silver coated overbraid is another source of voltage difference (Fig. 8),

- The mounting bracket chrome-plated pin in contact with an aluminum bracket creating a voltage source around the loop in the bracket (Fig. 9),

- The metallic backshell and grounding plug allowing eddy currents to form near the magnetometer (Fig. 10),

- Two thermal blanket copper ground wires emanating from the thermal blanket in contact with aluminized Kapton inner layers of the sensor multi-layer insulation creating a possible current loop.

The investigation team identified as a potential source for magnetic contamination the inadvertent application of Germanium Black Kapton (GBK) tape with nickel coated particles embedded in the tape's adhesive for charge dissipation instead of non-dissipative tape as required. The tape remained on the blankets for about three days before the tape was removed and the correct GBK tape applied. Per normal process for multi-layer blankets, the blankets were not cleaned after removal of the initial tape application so it is likely residual adhesive with nickel coated particles remained on the blanket.

Additionally, subsequent ground testing of the followon GOES-S magnetometer revealed cable lengths used on the spacecraft were different from those used in testing performed by the magnetometer manufacturer, resulting in zero offsets different than those determined in ground testing. This is likely a contributing root cause to the large zero offsets observed at boom deployment and subsequent calibrations.

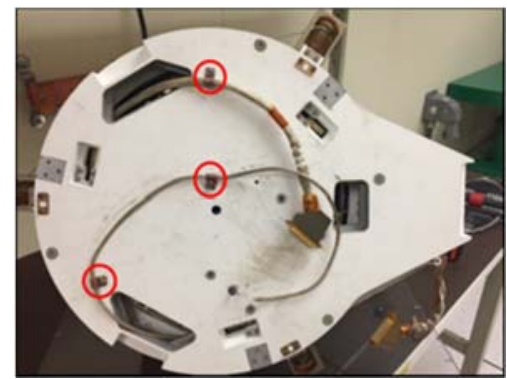

Figure 8 Copper wrapped P-clamps in contact with harness and VDA creating possible current loop

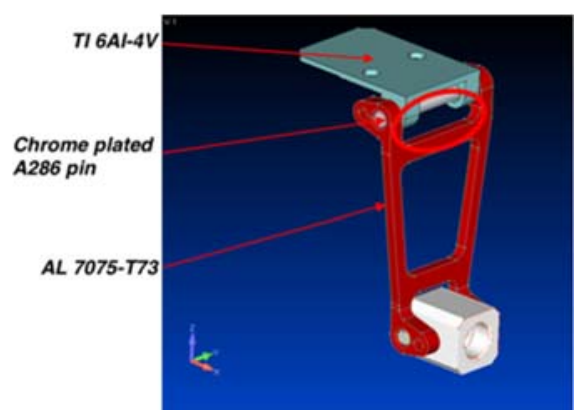

Figure 9 Mounting bracket with chrome in contact with Aluminum creating possible current loop

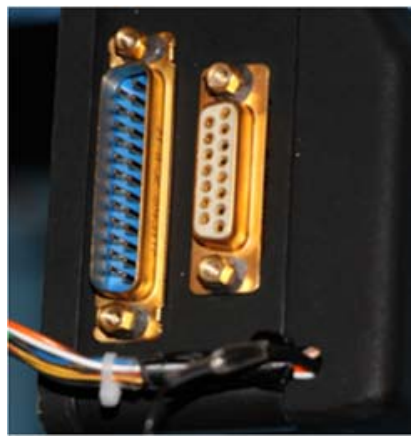

Figure 10 Metallic Backshells

\section{COMPONENT TESTING AND ANALYSIS}

Several ground tests were performed to attempt to definitize root cause. The focus was initially on the VDA used as a bonding material for the white paint. The concern is the VDA will either allow eddy currents to form as described in [5] or allow a current loop to form generated by the dissimilar material between the copper covering the p-clamps and the VDA or harness overbraid.

\subsection{VDA on Plate testing}

To test the theory that either the eddy currents are forming in the VDA or a current loop is formed along the harness braid and VDA, exact duplicates of the inboard and outboard plates were manufactured. This allowed the tests to be performed with as flight-like hardware as practical.

To test for thermo-magnetism as a result of eddy currents, one side of the plate was either heated with a heat gun or cooled with liquid nitrogen to generate a substantial thermal gradient across the plate. Thermocouples were attached to the plate to measure the thermal gradient. The plate was then moved from the far field to the near field of a test magnetometer. With thermal gradients as much as $80{ }^{\circ} \mathrm{C}$, no magnetic field emanating from the plate could be detected.

To test for the existence of a current loop along the harness overbraid and VDA, a section of harness was mounted to copper-wrapped p-clamps attached to the 
plate replicating the flight configuration. One P-clamp was heated with a heat gun to generate a thermal gradient between the junctions (Copper/Silver or Copper/Aluminum). Once again, no magnetic field was generated. From additional testing of different configurations including replacing the plate with an aluminum foil covered block, the investigation team concluded the VDA is too thin, approximately $600 \mu \mathrm{in}$, to provide an appreciable conductive path between the $\mathrm{P}$ clamps.

\subsection{Mounting Bracket Analysis}

An audit of all materials near the magnetometers looking for dissimilar metals in contact found the mounting bracket of the inboard plate has a chromium plated pin in contact with aluminum and titanium. Since the Seebeck coefficient for chromium and aluminum is 20.1 and -2.9 $\mu \mathrm{V} / \mathrm{K}[6][7]$, there is a possibility for large voltage with relatively small temperature gradient. Treating the loop in Figure 9 as circular, Eq. 1 is used to estimate the magnetitic field as a function of temperature gradient.

$$
B=\frac{\mu_{0}}{4 \pi} \frac{2 \pi R^{2} I}{\left(z^{2}+R^{2}\right)^{3 / 2}}
$$

The distance from the bracket to the magnetometer is only $0.076 \mathrm{~m}$. However, the effective radius ( $\mathrm{R}$ in eq. 1) for the loop area is only $5.5 \mathrm{E}-3 \mathrm{~m}$. This yields a field at the magnetometer of $0.26 \mathrm{nT} / \mathrm{K}$. To be the primary contributor of the magnetic contamination during shadow transition, the thermal gradient needs to be $\sim 77 \mathrm{~K}$. Given the thermal conduction and thermal mass of the bracket, it is unlikely such a gradient can be achieved.

\subsection{Thermal Blanket Grounding Wires}

The thermal blanket has two grounding wires emanating from either end of the blanket. Since the layers of the blanket are aluminum sheet and the wire is copper, there is possibility of thermoelectric effect. Similarly to the VDA testing, a spare blanket was manufactured and tested for a magnetic field when exposed to heating on one side of the blanket. Once again, no measurable field was observed.

\subsection{GBK Tape Testing}

To test for the possibility of residual tape adhesive with nickel coated particles being a magnetic contamination source, pieces of tape were removed from flight stock and measured for magnetic field. While the measured field and direction varied from piece to piece and with size, fields in excess of 60nT were observed. Seven pieces were then applied to blanket material and left for three days before removing the tape as shown in Fig. 11. The resulting magnetic field was approximately $5 \mathrm{nT}$.

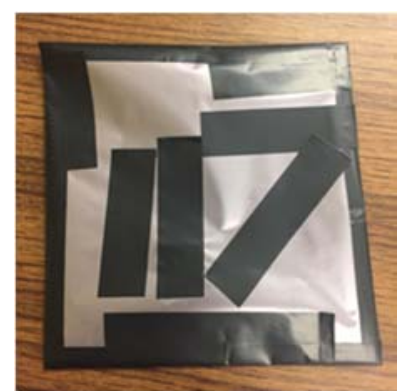

Figure 11 GBK Dissipative Tape Applied to Blanket Material

While this finding partly explains the large static bias, it does not explain the varying field. The outer layer of a thermal blanket will vary between $-175^{\circ} \mathrm{C}$ and $75^{\circ} \mathrm{C}$, as shown in Fig. 12, which will cause the magnetic field density to vary as the tape on the blanket goes in and out of shadow.

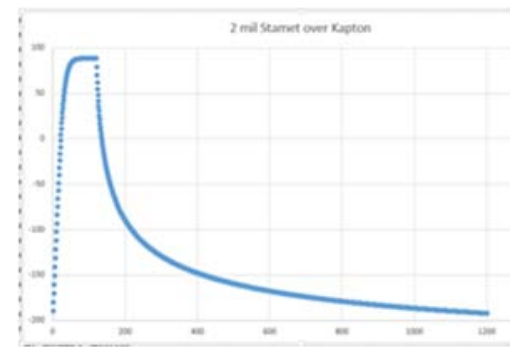

Figure 12 Predicted Temperature of Outer Layer of Thermal Blanket During Entry and Exit of Shadow.

In order to bound the expected change in magnetic field due to temperature change, a piece of tape was sandwiched between foam and dunked into liquid nitrogen until it was colder than $-130{ }^{\circ} \mathrm{C}$. It was then placed on a polyetherimide cover with a test magnetometer thermally isolated underneath as shown in Fig. 13. The magnetic field from the tape was measured as the foam/tape sandwich was allowed to warm in ambient air. Control cases were run without tape to demonstrate the magnetometer was not being thermally influenced.

The change in the field over the test temperature was approximately $0.05 \mathrm{nT} / \mathrm{C}$. For the cited temperature range, a change in field of approximately $12 \mathrm{nT}$ is possible. 


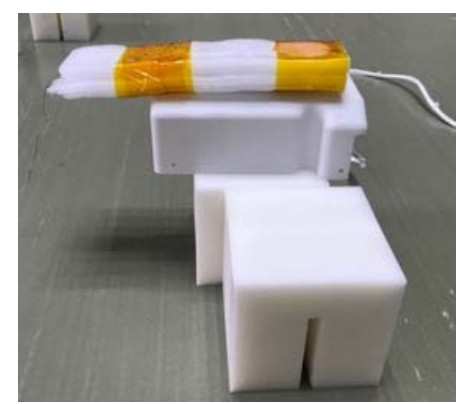

Figure 13 Test Setup to Measure Magnetic Field with Changing Temperature of Tape Sample.

\subsection{GOES-S Spacecraft Testing}

As part of the lessons learned and root cause investigation, a shield can test was added to the GOES-S spacecraft testing. This involved removing the magnetometers from the stowed boom and placing them in shield cans to measure noise and zero offset in ambient conditions. The zero offsets were measured by manually rotating the sensors in the shield cans.

The measured zero offsets in all three axes were significantly different, up to $12 \mathrm{nTs}$, from those measured by the magnetometer manufacturer. Investigation determined that the magnetometer manufacturer was testing and tuning the magnetometers in a cable configuration inconsistent with the flight configuration. Since the same cable configuration was used for GOES$\mathrm{R}$, this inconsistency accounts for at least some of the unexpected zero offset observed on orbit. Given the build-to-build variation in the magnetometers, it is not possible to determine how much the incorrect cable configuration contributes to the unexpected GOES-R zero offset

\section{THERMAL REDESIGN}

During the course of the anomaly investigation it was determined that insufficient thermal control requirements result in large swings in the bobbin temperature as shown in Fig. 14. The magnetometer ground processing algorithm corrects for temperature influences on the zero offsets, however such large temperature swings can exacerbate errors in the pre-launch determined compensation coefficients, or create unexpected gradients in the bobbins that change the calibration. Such large temperature swings are likely major contributor to the errors in the outboard magnetometer shown in Fig. 4.

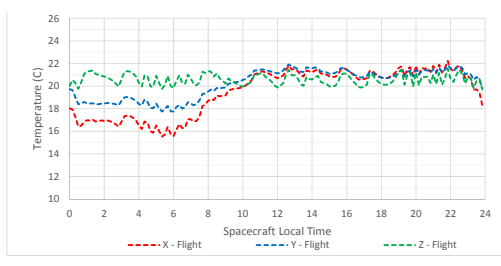

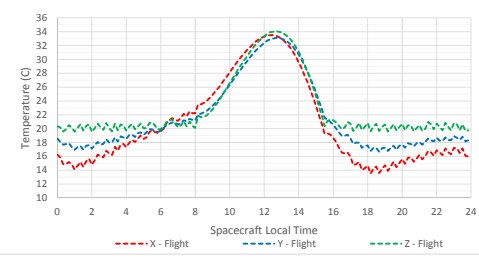

Figure 14 Inboard (top) and Outboard (bottom) Bobbin Diurnal Temperatures

Since the temperature swings were well outside what was predicted by the thermal model, a standalone (magnetometer sensor mounted on flight plate) thermal balance (TB) test was defined and performed to create a correlated model. During the TB test several design changes were tested, including the addition of another blanket over the existing blanket, the addition of blankets over the harnesses, and the addition of a heater and control thermistor to the harness adjacent to the sensor unit to reduce heat loss through the harness. Fig. 15 shows the test set up for the TB test.
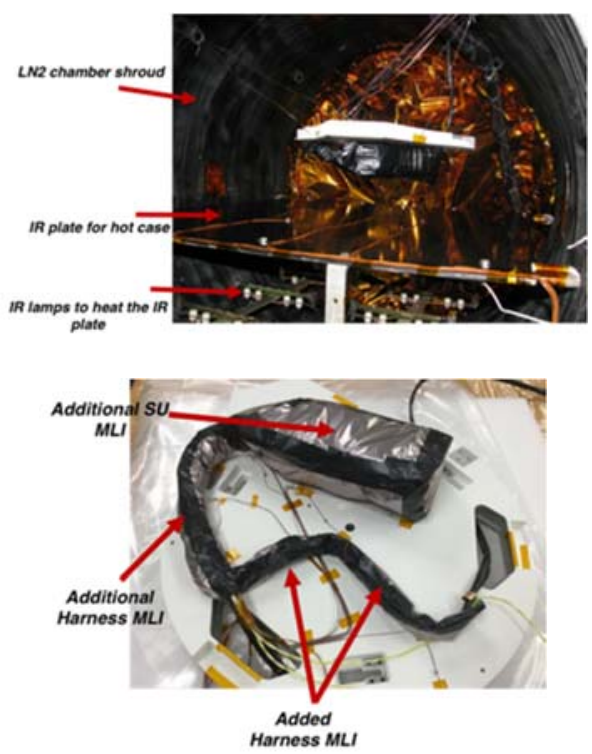

Figure 15 Thermal Balance Test Setup with Thermal Changes.

The TB test demonstrated the proposed changes would significantly improve the thermal control of the magnetometer. Tab. 1 shows the seasonal gradients of the bobbins for the GOES-16 design and for the redesign.

Table 1 Bobbin Gradients Before and After Thermal

\begin{tabular}{|c|c|c|c|c|}
\hline & & Chang & ges & \\
\hline & & \begin{tabular}{|c|} 
Phase 1 \\
Hot-Cold
\end{tabular} & $\begin{array}{c}\text { Phase } 2 \text { 2 } 35^{\circ} \mathrm{C} \\
\text { Hot Balance } 3 \text {. Cold Balance } 10\end{array}$ & Improvement \\
\hline & X Bobbin Gradient ("C) & -3.1 & Hot batance 3 - Cola batance iv & \begin{tabular}{|c|} 
\\
48.4 \\
\end{tabular} \\
\hline & Y Bobbin Gradient ("C) & .8 .0 & .2 .0 & 75.0 \\
\hline botolins & 2 Bobbin Gradient ("C) & -2.8 & -2.1 & 25.0 \\
\hline & Across All Bobbin Gradient ("C) & -14.8 & -5.5 & 62.8 \\
\hline
\end{tabular}




\section{MAGNETOMETER ACCOMMODATION CHANGES}

Although there is strong indication the root cause of the anomalous signature of the GOES-R magnetometers is workmanship related (tuning and calibrating using incorrect cable configuration, and inadvertently adding magnetic contamination to the blankets), the investigation team decided the best course of action was to eliminate all potential sources of thermo-electric effect and current loops. This included:

- $\quad$ scoring the inboard and outboard VDA-coated mounting plates to decrease the potential of thermally induced eddy currents

- replacing of the copper tape on the p-clamps with non-conductive GBK tape to prevent electrical contact between the harness and the VDA

- $\quad$ isolating the multiple harnesses from each other to prevent the possibility of current loop through the metallic braid

- replacing the chrome-plated pin of the plate mounting bracket with a ceramic pin to prevent the Seebeck effect and path for current loops

- replacing the metallic backshell and grounding plug cover with non-conductive polymeric 3-D printed parts to eliminate any metallic material near the sensors.

Whatever the root cause of the anomalous signature on GOES-16, the steps taken greatly improved the performance of the magnetometers on GOES-17, which was launched March 1, 2018. Fig. 16 shows the difference in the $y$-axis inboard and outboard magnetometers during a shadowing period. The large swings evident in Fig. 2 are no longer present and the diurnal variation is greatly reduced.

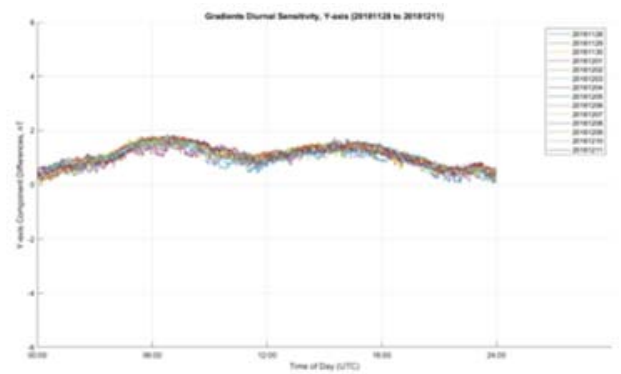

Figure 16. GOES-17 daily trend of difference between inboard and outboard over a 2 week period showing no anomalous signature

\section{CONCLUSION}

The root cause investigation and accommodation redesign has led to several lessons learned in the design, observatory integration, and handling of science grade magnetometers:
1. Stringent magnetic screening and monitoring needs to be in place and followed at all times. All material used near the magnetometer needs to be screened and tagged as magnetically clean. Reliance on part numbers and kitting is insufficient.

2. Calibration of the sensors needs to be performed at the highest level of accuracy if there is any chance of thermal drift or thermal gradients. This includes measuring in a flightlike thermal environment.

3. System level testing at the spacecraft needs to include the ability to trend the zero offsets through the integration and test program.

4. The accommodation design must eliminate the possibility of current loops, including in thermal blankets and harnassing.

5. Adequate thermal requirements need to established to maintain minimal temperature swings across the bobbins.

6. Instrument level thermal balance is needed since spacecraft level thermal balance is insufficient for the accuracy required.

\section{REFERENCES}

1. Carter, D., Freesland, D., Tadikonda, S., Kronenwetter J., Todirita, M., Dahya J., \& Chu D. (2016). Correcting GOES-R Magnetometer Data for Stray Fields. In Proc. 2016 ESA 'Workshop on Aerospace EMC', (Ed. L. Ouwehand), ESA SP-738 (CDROM), ESA Communications, ESTEC, Noordwijk, The Netherlands

2. Carter, D., Early, D., Kronenwetter J., Grotenhuis, M., Schnurr, R., Todirita, M., (2018). GOES-16 Magnetometers Anomaly Solar-Angle Based Characterization \& Correction. In Proc AMS Annual Meeting '15th Annual Symposium on New Generation Operational Environmental Satellite Systems' (Eds. I. Csiszar \& J. Forsythe) (CD-ROM).

3. Rich, F., Lotoanlu P., Califf S., Carter, D., Kronenwetter J., (2018) Comparison of Magnetic Field Measurements Across the Geostationary Operational Environmental Satellites (GOES). In Proc AGU Fall Meeting 'SPA-Magnetospheric Physics' (CD-ROM).

4. Vasiliev, B.V. (2014). The New Thermo-magnetic Effect in Metals. Universal Journal of Physics and Application 2(4), 221-225.

5. Acuña, M.H. (2004). The design, construction and test of magnetically clean spacecraft A practical guide. NASA/GSFC internal report, Rev 3.0.

6. Hust, J. G., Weitzel D. H., \& Powell, R. L. (1971). Thermal Conductivity, Electrical Resistivity, and 
Thermopower of Aerospace Alloys From 4 to 300

$\mathrm{K}$. Journal of Research of the National Bureau of Standards, Section A. 75, 269-277

7. Morozova, N. V., Shchennikov, V. V., \& Ovsyannikov S. V., (2015). Features and regularities in behavior of thermoelectric properties of rare-earth, transition, and other metals under high pressure up to $20 \mathrm{GPa}$. Journal of Applied Physics 118 (22), 225901 .

\section{ACKNOWLEDGMENTS}

The authors wish to thank our colleague Cindy Merrow for review and comments of this paper. 